\title{
GENERALIZED STEENROD-HOPF INVARIANTS FOR STABLE HOMOTOPY THEORY
}

\author{
WARREN M. KRUEGER
}

\begin{abstract}
In his paper On the groups $J(X)$. IV, Adams suggested that one might try to continue his $d$ and $e$ invariants to a sequence of higher homotopy invariants, each defined upon the vanishing of its predecessors and each taking its value in a certain Ext group. Recently he pointed out the efficacy of relocating his $d$ and $e$ invariants in Ext groups formed over a certain abelian category of comodules. It is the purpose of this note to carry out the program suggested above in a setting of the sort just mentioned. More specifically, for each homology theory which is representable by a comutative ring spectrum and whose ring of cooperations is flat over the coefficient ring, a sequence of higher homotopy invariants is constructed whose first term is Adams' $e$ invariant for this theory.
\end{abstract}

1. Notation and statement of results. In this section some notation is fixed and the main results of this note are given. Their proofs are deferred until $\S 3$.

All spectra and maps considered here are assumed to reside in Boardman's stable category.

From now on, $E$ shall denote a commutative ring spectrum with unit $i: S^{0} \rightarrow E$. It shall be further assumed that $E_{*}(E)$ is flat as a left $E_{*}\left(S^{0}\right)$ module. Then $E_{*}(E)$ is a Hopf algebra over which $E_{*}(X)$ is a left comodule for any spectrum $X$. All Hom and Ext groups found here are formed relative to the abelian category of left $E_{*}(E)$ comodules.

Let $f: X \rightarrow Y$ be a map whose domain satisfies the isomorphism condition: for any spectrum $W$, the correspondence

$$
[X, E \wedge W]_{*} \rightarrow \operatorname{Hom}_{E_{*}(E)}^{*}\left(E_{*}(X), E_{*}(E \wedge W)\right),
$$

given by $[g] \rightarrow g_{*}$, is an isomorphism. This condition obtains, for example, if $X$ satisfies the hypotheses of Proposition 17 of [2].

Received by the editors November 27, 1972.

AMS (MOS) subject classifications (1970). Primary 55E25; Secondary 55E45, 55G20.

Key words and phrases. Stable homotopy theory, spectrum, coalgebra, comodule, homology operation, exact couple, canonical injective resolution, extended comodule, unitary spectrum.

(c) American Mathematical Society 1973 
For stable maps $f: X \rightarrow Y$ of the sort just described for which also $f_{*}: E_{*}(X) \rightarrow E(Y)$ is zero, higher order functional homology operations are constructed after the manner of Peterson from exact couples owing to Adams. These operations evaluated on a certain fundamental class yield subsets

$$
I_{n}(f) \subset \operatorname{Ext}_{E_{*}(E)}^{n, n}\left(E_{*}(X), E_{*}(Y)\right),
$$

which are invariants of the homotopy class of $f$. The set $I_{n}(f)$, defined when $I_{k}(f)=0$ for $k<n$, is called the $n$th generalized Steenrod-Hopf invariant of $f$ with respect to $E$.

The values of $I_{1}$ and $I_{2}$ are given in terms of more familiar operations in the next two propositions. In particular, the first of these identifies $I_{1}$ as Adams' $e$ invariant up to sign.

Proposition 1. When $I_{1}(f)$ is defined, $I_{1}(f)=-e(f)$, where $e(f)$ is Adams' $e$ invariant of $[\mathbf{1}, p .27]$.

The second proposition might be considered, from a heuristic point of view, as a consequence of Peterson's composition theorem [5, p. 208]. Proposition 2 is proved, however, without reference to Peterson's theorem, as his proof of that result does not apply in this setting.

Proposition 2. Suppose $f=h \circ g$ and $I_{1}(g)$ and $I_{1}(h)$ are defined. Then (a) $I_{2}(f)$ is defined; and

(b) $-\left(I_{1}(h) \cdot I_{1}(g)\right) \in I_{2}(f)$, where "." denotes composition in Ext.

The next proposition specifies the differential in the Adams exact couple by a formula which is useful in the calculation of the invariants $I_{n}$. Its precise statement, being somewhat technical, is given in Proposition 3 of the next section.

Finally the above results are illustrated in some computations involving complex $K$-homology. While more comprehensive computations are possible by the same methods, the ones given here serve to typify the basic ingredients of the methods in a clear way. A summary of these computations is given in

Proposition 4. Let $K$ be the unitary spectrum.

(a) $\operatorname{Ext}_{\left.K_{*}^{1}, K^{\prime}\right)}\left(K_{*}\left(S^{0}\right), K_{*}\left(S^{0}\right)\right)$ is isomorphic to $Z_{2}$ with generator $I_{1}(\eta)$, where $\eta \in\left[S^{0}, S^{0}\right]_{1}$ is the class of the stable Hopf map.

(b) $I_{2}\left(\eta^{2}\right)$ is defined and nonzero in $\operatorname{Ext}_{K_{*}^{2},(K)}^{24}$

2. The Adams exact couple and homotopy invariants. First a bit of notation: If $f: X \rightarrow Y$ is a map, then its associated mapping triangle is 
denoted by

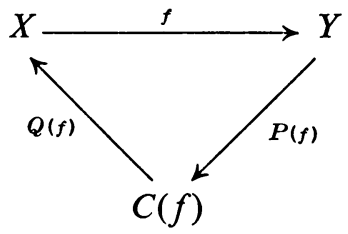

where $C(f)$ is the mapping cone and $P(f)$ and $Q(f)$ are the obvious maps.

The Adams exact couple is obtained from the filtration construction of [2, p. 54]. For later convenience, this construction is recounted here in slightly different form than in [2]. Let $C(i)$ be the mapping cone of $i: S^{0} \rightarrow E$ and, for any spectrum $W$, let $W^{k}$ denote the $k$-fold smash of $W$ with itself. For any spectrum $Y$, define $Y_{k}$ to be $(C(i))^{k} \wedge Y, Z_{k}$ to be $E \wedge Y_{k}, i_{k}$ to be $i \wedge Y_{k}, j_{k}$ to be $P(i) \wedge Y_{k}$ and $p_{k}$ to be $Q(i) \wedge Y_{k}$. The resulting diagram

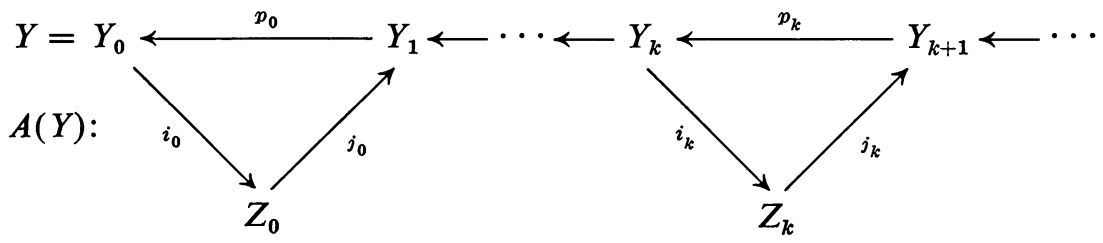

is called the Adams filtration for $Y$.

Applications of the functor $\left[X,{ }_{-}\right]_{*}$ to $A(Y)$ yields an exact couple $\mathscr{A}(X, Y)$ in which $A^{s, t}=\left[X, Y_{s}\right]_{t}, C^{s, t}=\left[X, Z_{s}\right]_{t}$ and the maps $f: A \rightarrow A$, $g: C \rightarrow A$ and $h: A \rightarrow C$ of bidegrees $(-1,-1),(0,0)$ and $(1,0)$ are induced by $p_{s}, i_{s}$, and $j_{s}$ respectively. This couple is called the Adams exact couple of $X$ and $Y$. In the event that $X$ satisfies the isomorphism condition, $\mathscr{A}(X, Y)$ has the $E_{2}$-term of its associated spectral sequence isomorphic to

$$
\operatorname{Ext}_{E_{*}(E)}^{* *}\left(E_{*}(X), E_{*}(Y)\right) \text {. }
$$

In addition, if $X=S^{0}$, the differential of $\mathscr{A}\left(S^{0}, Y\right)$ satisfies a useful formula which is recorded in

Proposition 3. The differential $d_{1}^{s, *}: \pi_{*}\left(Z_{s}\right) \rightarrow \pi_{*}\left(Z_{s+1}\right)$ satisfies the formula

$$
v^{-1} d_{1}^{s, *}=\left(j_{0 *} \otimes 1\right)(c \otimes 1) \psi_{Y_{s}},
$$

where $v$ is the external product for $E_{*}$ homology, $j_{0}: E \rightarrow C(i), c$ is the canonical anti-automorphism for $E_{*}(E)$ and

$$
\psi_{Y_{s}}: E_{*}\left(Y_{s}\right) \rightarrow E_{*}(E) \otimes_{E_{*}\left(s^{0}\right)} E_{*}\left(Y_{s}\right)
$$

is the coaction map. 
The generalized Steenrod-Hopf invariants, which can be introduced now, are functional homology operations evaluated on a certain fundamental class. These operations arise by the method of Peterson $[5, \S 5]$ applied to Adams' exact couples.

Specifically, let $f: X \rightarrow Y$ be a map whose domain satisfies the isomorphism condition and for which $f_{*} E_{*}(X) \rightarrow E_{*}(Y)$ is zero. In $\S 5$ of [4], let $\left(A^{\prime}, C^{\prime}\right),(A, C)$ and $\left(A^{\prime \prime}, C^{\prime \prime}\right)$ be the couples $\mathscr{A}(X, Y), \mathscr{A}(X, C(f))$ and $\mathscr{A}(X, X)$ respectively and $i$ and $j$ be the maps of bidegree $(0,0)$ and $(0,-1)$ induced by $P(f)$ and $Q(f)$ respectively. Further, take $\iota \in[X, E \wedge X]_{0}$ to be the class of $i_{0}$ in $A(X)$. Then $d_{k}\{l\}=\left\{i_{k *} f^{-(k-1)} j_{0 *}(\iota)\right\}=0$ for all $k$.

Now if $\Delta_{1}\{l\}=0, \cdots, \Delta_{n-1}\{l\}=0$, then $\Delta_{n}\{l\}$ is defined. This class is called the $n$th generalized Steenrod-Hopf invariant of $f$ with respect to $E$ and is denoted by $I_{n}(f)$. From its definition, it is easy to see that this class is both an invariant of $f$ up to homotopy and a subset of

$$
\operatorname{Ext}_{E *(E)}^{n, n}\left(E_{*}(X), E_{*}(Y)\right) .
$$

As an example, if $E=H\left(Z_{2}\right)$, the Eilenberg-Mac Lane spectrum for $\boldsymbol{Z}_{2}$, and $f$ is an element of a stem, then $I_{1}(f)$ is the stable mod 2 Hopf invariant of $f$.

It shall be useful later to note that Adams' $e$ invariant can be formulated in terms of $\mathscr{A}(X, Y)$ provided $X$ satisfies the isomorphism condition. Since $f_{*}=0, i_{0} \circ f \simeq 0$; so there is a map $f: X \rightarrow Y_{1}$ of degree +1 for which $f \simeq p_{0} \circ f$. The homomorphism

determines a class

$$
\left(i_{1} \tilde{f}\right)_{*} \in \operatorname{Hom}_{E_{*}(E)}^{1}\left(E_{*}(X), E_{*}\left(Z_{1}\right)\right)
$$

$$
e^{\prime}(f) \in \operatorname{Ext}_{E_{*}(E)}^{1.1}\left(E_{*}(X), E_{*}(Y)\right)
$$

which clearly depends upon only the homotopy class of $f$. The class $e^{\prime}(f)$ corresponds to Adams' invariant $e(f)$ under the isomorphism which identifies the $\operatorname{Ext}^{1}$ group defined from resolutions with the $\mathrm{Ext}^{1}$ group defined by the classification of short exact sequences.

3. Proofs. The proofs of Propositions 1 through 4 are given in this section.

The proofs of the first two propositions use a certain geometric fact which is presumably well known but does not seem to be written down anywhere. It is given here without proof as

PropCSITION 5. Suppose given two factorizations of a null-homotopic map, i.e., $h_{1} g_{1}=h_{2} g_{2} \simeq 0: W \rightarrow Y$. Then there exist maps $\tilde{g}_{\varepsilon}: W \rightarrow C\left(h_{\varepsilon}\right)$ of degree $+1, \varepsilon=1,2$, such that

(i) $Q\left(h_{1}\right) \tilde{g}_{1} \simeq-g_{1}$ and $Q\left(h_{2}\right) \tilde{g}_{2} \simeq g_{2}$; 
(ii) the maps $i_{1} \tilde{g}_{1}$ and $i_{2} \tilde{g}_{2}$ are homotopic, where $i_{\varepsilon}: C\left(h_{\varepsilon}\right) \rightarrow C\left(h_{1} \vee h_{2}\right)$ is the inclusion, for $\varepsilon=1,2$.

The idea in the proof of 1 and 2 is to advantageously use the choices permissible in the definitions of $I_{1}$ and $I_{2}$ to produce a suitable representative of each.

Proof of Proposition 1. By definition,

$$
I_{1}(f)=\left\{(E \wedge C(i) \wedge P(f))_{*}^{-1} d_{1}(E \wedge Q(f))_{*}^{-1}(\iota)\right\},
$$

where $d_{1}=i_{1 *} j_{0 *}$ in the couple $\mathscr{A}(X, C(f))$. The map $(i \wedge Y) f=(E \wedge f)(i \wedge X)$ is null-homotopic, since $f_{*}=0$. By Proposition 5 , there are classes $\tilde{\imath} \in$ $[X, E \wedge C(f)]_{1}$ and $[\tilde{f}] \in[X, C(i) \wedge Y]_{1}$ so that $(E \wedge Q(f))_{*}(\tilde{\iota})=\iota, p_{0 *}[\tilde{f}]=$ $-[f]$ and $(C(i) \wedge P(f))_{*}\left(\left[\tilde{f}^{\tilde{f}}\right]\right)=j_{0 *}(\tilde{\imath})$. Thus on one hand, $i_{1 *}([\tilde{f}])$ represents $I_{1}(f)$ and, on the other hand, it represents $-e^{\prime}(f)$. As $e^{\prime}(f)=e(f)$, the proof is complete.

Proof of Proposition 2. According to its definition

$$
I_{2}(h g)=\left\{\left[E \wedge\left(C(i)^{2} \wedge P(h g)\right]_{*}^{-1} d_{2}(E \wedge Q(h g))_{*}^{-1}(\iota)\right\},\right.
$$

where $d_{2}=i_{2 *} p_{1 *}^{-1} j_{0 *}$ in the couple $\mathscr{A}(X, C(h g))$. By the methods of the preceding proof, there are classes $\tilde{\imath} \in[X, E \wedge C(g)]_{1}$ and $[\tilde{g}] \in[X, C(i) \wedge W]_{1}$ so that $(E \wedge Q(g))_{*}(\imath)=\iota \in[X, E \wedge X]_{0}, p_{0 *}([\tilde{g}])=-[g]$ and

$$
(C(i) \wedge P(g))_{*}([\tilde{g}])=j_{0 *}([\tilde{\imath}]) .
$$

There is also a map $\hat{h}: C(g) \rightarrow C(h g)$ for which $Q(h g) \hat{h}=Q(g)$. Then $\alpha=(E \wedge \hat{h})_{*}(\tilde{\iota}) \in[X, E \wedge C(h g)]_{1}$ and $(E \wedge Q(h g))_{*}(\alpha)=\iota$. Further $j_{0 *}(\alpha)=$ $(C(i) \wedge P(h g))_{*}([(C(i) \wedge h) \tilde{g}])$.

Now let $\tilde{h}: W \rightarrow C(i) \wedge Y$ be such that $p_{0 *}([\tilde{h}])=[h]$. Then $i_{2 *}([(C(i) \wedge \tilde{h}) \tilde{g}])$ represents $-I_{1}(h) \cdot I_{1}(g)$. Further $p_{1 *}([(C(i) \wedge \tilde{h}) \tilde{g}])=[\tilde{h} g]$. As both $[(C(i) \wedge h) \tilde{g}]$ and $[\tilde{h} g]$ project to $[h g]$ under $p_{0 *}$, there is a class $\beta \in$ $[X, E \wedge Y]_{1}$ so that $j_{0 *}(\beta)=[\tilde{h} g]-[(C(i) \wedge h) \tilde{g}]$. Now $\alpha+(E \wedge P(h g))_{*}(\beta)$ projects to $\iota$ under $(E \wedge Q(h g))_{*}$ and projects to $[(C(i) \wedge P(h g)) \tilde{h} g]$ under $j_{0 *}$. This implies that $i_{2 *}([(C(i) \wedge \tilde{h}) \tilde{g}]) \in I_{2}(h g)$ and the proof is complete.

In Proposition 3, the formula satisfied by the Adams differential is a consequence of a more general algebraic result. The algebraic setting for this result is as follows.

Let $K$ be a commutative ring with unit, $\Lambda$ a $K$-bimodule which is an augumented Hopf algebra with canonical anti-automorphism $c$ and $A$ a left $K$-module which is a left $\Lambda$ comodule with structure map $\psi_{A}: A \rightarrow$ $\Lambda \otimes_{K} A$. Further, let $\psi_{A}: A \rightarrow X$ be the canonical injective resolution of $A$ (for definition and notation see p. 208 of [4]) and let $K^{1}$ be $\operatorname{coker}\left\{\eta_{L}: K \rightarrow \Lambda\right\}$, where $\eta_{L}$ is the left unit of $\Lambda$. Note that $K^{1}$ is a left 
$K$-module. Now suppose given for each $p$, an isomorphism

so that

$$
v_{p}: K^{1} \otimes_{K} A^{p} \rightarrow A^{p+1}
$$

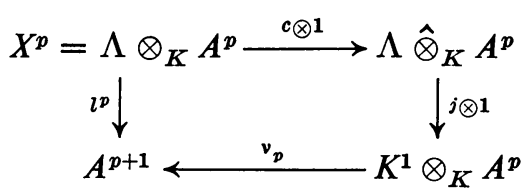

commutes, where $j: \Lambda \rightarrow K$ is the quotient and " $\hat{\otimes}$ " is used to indicate that the tensor product is formed using the left action of $K$ on $\Lambda$, instead of the usual right action. Of course, $K^{1} \otimes_{K} A^{p}$ is the tensor product of left $K$-modules.

Proposition 6. The complex $\left\{\operatorname{Hom}_{\Lambda}\left(K, X^{p}\right), \operatorname{Hom}_{\Lambda}\left(K, d^{p}\right)\right\}$ is chain isomorphic to the complex $\left\{A^{p}, d_{p}\right\}$ in which the differential $d_{p}$ is given by the formula

$$
v_{p}^{-1} d_{p}=(j \otimes 1)(c \otimes 1) \psi_{A}^{p} .
$$

Proof. From the properties of extended comodules,

$$
A^{p} \simeq \operatorname{Hom}_{K}\left(K, A^{p}\right) \simeq \operatorname{Hom}_{\Lambda}\left(K, \Lambda \otimes_{K} A^{p}\right)=\operatorname{Hom}_{\Lambda}\left(K, X^{p}\right) .
$$

To complete the proof, it suffices to show that the composite

$$
d_{p}^{\prime}: A^{p} \simeq \operatorname{Hom}_{\Lambda}\left(K, X^{p}\right) \rightarrow \operatorname{Hom}_{\Lambda}\left(K, X^{p+1}\right) \simeq A^{p+1}
$$

satisfies the formula giving $d_{p}$. From its definition $d_{p}^{\prime}(a)=l^{p}\left(\eta_{L}(1) \otimes a\right)$, $a \in A^{p}$. As $l^{p}$ annihilates $\operatorname{im} \psi_{A^{p}}$,

$$
d_{p}^{\prime}(a)=l^{p}\left(\eta_{L}(1) \otimes a+\psi_{A^{p}}(a)\right) .
$$

Now

$$
\begin{aligned}
v_{p}^{-1} d_{p}^{\prime}(a) & =v_{p}^{-1} l^{p}\left(\eta_{L}(1) \otimes a+\psi_{A^{x}}(a)\right) \\
& =(j \otimes 1)(c \otimes 1)\left(\eta_{L}(1) \otimes a+\psi_{A^{p}}(a)\right) \\
& =\left(j c \eta_{L}(1) \otimes a\right)+(j \otimes 1)(c \otimes 1) \psi_{A^{p}}(a) \\
& =(j \otimes 1)(c \otimes 1) \psi_{A^{p}}(a), \quad a \in A^{p} .
\end{aligned}
$$

Proof of Proposition 3. Relative to the above algebra, set $K=E_{*}\left(S^{0}\right)$, $\Lambda=E_{*}(E), K^{1}=E_{*}(C(i)), v_{p}=$ the external homology product for $E_{*}$ (see [2, p. 58]) and $A=E_{*}(Y)$. Since $i_{p *}=m \psi_{Y_{p}}: E_{*}\left(Y_{p}\right) \rightarrow E_{*}\left(E \wedge Y_{p}\right)$ and $l^{p}=j_{n *} m: E_{*}(E) \otimes_{E_{*}\left(S^{0}\right)} E_{*}\left(Y_{p}\right) \rightarrow E_{*}\left(Y_{p+1}\right)$, the resolution

$$
0 \rightarrow E_{*}(Y) \rightarrow E_{*}\left(Z_{0}\right) \rightarrow \cdots
$$

obtained from $A(Y)$ is nothing but the canonical injective resolution of $E_{*}(Y)$, where the isomorphism $m: E_{*}(E) \otimes_{E_{*}\left(S^{0}\right)} E_{*}\left(Y_{p}\right) \rightarrow E_{*}\left(E \wedge Y_{p}\right)$ is the 
product defined in [2, p. 58]. The proposition now follows from Proposition 6 , since the correspondence $\pi_{*}\left(Z_{p}\right) \rightarrow \operatorname{Hom}_{E_{*}(E)}^{*}\left(E_{*}\left(S^{0}\right), E_{*}\left(Z_{p}\right)\right)$, given by $[f] \mapsto f_{*}$, is a chain isomorphism.

Proof of Proposition 4. (a) The group $\operatorname{Ext}_{K_{*}(K)}^{1,2}\left(K_{*}\left(S^{0}\right), K_{*}\left(S^{0}\right)\right)$ is isomorphic to $\operatorname{ker} d_{1}^{1,2} / \mathrm{im} d_{1}^{0,2}$. By Proposition 3 and Theorem (2.3) of [3], $\operatorname{ker} d_{1}^{1,2}=\{r(v-u) \mid r$ is a suitable rational $\}$. The integrality condition (2.4) of [3] requires that $r \equiv \frac{1}{2}(\bmod 1)$. On the other hand $\operatorname{im} d_{1}^{0,2}=\{n(v-u) \mid n \in Z\}$. Thus $\operatorname{Ext}_{K_{*}(K)}^{1,2}$ is isomorphic to the subgroup of order 2 of coker $\dot{d}_{1}^{0,2}$ generated by $\frac{1}{2}(v-u)$.

To show that $I_{1}(\eta)$ assumes the value $\frac{1}{2}(v-u)$, one simply applies the definition of $I_{1}$. The mapping cone of $\eta$ is $\Sigma^{-2} C P^{2}$ in the stable category. Let $i \in K_{2}\left(\Sigma^{-2} C P^{2}\right)$ be the obvious element which projects to $\iota \in K_{1}\left(S^{1}\right)$ and $\iota_{0} \in K_{0}\left(\Sigma^{-2} C P^{2}\right)$ be the image under $S^{0} \rightarrow \Sigma^{-2} C P^{2}$ of the obvious element of $K_{0}\left(S^{0}\right)$. Then $v^{-1} d_{1}(\tilde{\imath})=\left(j_{0 *} \otimes 1\right)(c \otimes 1) \psi_{\Sigma^{-2} C P^{2}}(\iota)=\left(j_{0 *} \otimes 1\right) \times$ $(c \otimes 1)\left(a \otimes \imath_{0}+1 \otimes \tilde{\imath}\right)=j_{0 *} c(a) \otimes \iota_{0}$, where $a \in K_{2}(K)$. It is not hard to show that $a$ is represented by $t^{2} \beta_{2} \in \tilde{K}_{4}(B U)$ with $B U$ regarded as the second term, $K_{2}$, of the spectrum $K$. When $B U$ is taken to be $K_{0}, t^{2} \beta_{2}$ projects to $p_{2}^{\prime}=\frac{1}{2} v(v-u)$ by $(6.13)$ of [3], so when $B U$ is taken to be $K_{2}, t^{2} \beta_{2}$ projects to $\frac{1}{2}(v-u)$ according to $(6.1)$ of [3]. Thus $I_{1}(\eta)=j_{0 *} c\left(\frac{1}{2}(v-u)\right)=\frac{1}{2}(u-v)=$ $\frac{1}{2}(v-u)$ in $\operatorname{Ext}_{K_{*}(K)}^{1,2}\left(K_{*}\left(S^{0}\right), K_{*}\left(S^{0}\right)\right)$.

(b) For dimensional reasons, the indeterminacy of $I_{2}\left(\eta^{2}\right)$ is zero, so that by Proposition $2, I_{2}\left(\eta^{2}\right)$ is the singleton $\left\{-I_{1}(\eta) \cdot I_{1}(\eta)\right\}$. That this class is nonzero in Ext ${ }^{2}$ can be shown by calculating with $d_{1}^{2,4}$ and $d_{1}^{1,4}$ and the integrality condition (2.4) of [3].

\section{REFERENCES}

1. J. F. Adams, On the groups $J(X)$. IV, Topology 5 (1966), 21-71. MR 33 \#6628.

2. - - Lectures on generalized cohomology, Category Theory, Homology Theory and their Applications, III (Battelle Inst. Conf., Seattle, Wash., 1968), Springer, New York, 1969, pp. 1-138. MR 40 \#4943.

3. J. F. Adams, A. S. Harris and R. M. Switzer, Hopf algebras of cooperations for real and complex K-theory, Proc. London Math. Soc. (3) 23 (1971), 385-408

4. S. Eilenberg and J. C. Moore, Homology and fibrations. I. Coalgebras, cotensor product and its derived functors, Comment. Math. Helv. 40 (1966), 199-236. MR 34 \#3579.

5. F. P. Peterson, Functional cohomology operations, Trans. Amer. Math. Soc. 86 (1957), 197-211. MR 21 \#4417.

Department of Mathematics, New Mexico State University, las Cruces, New MeXICO 88001 